\title{
A Simple and Green Protocol for 2H-Indazolo[2,1-b]phthalazine-triones Using Grinding Method
}

\author{
Xiao-chuan Jia, ${ }^{1}$ Jing Li, ${ }^{1}$ Yu Ding, ${ }^{1}$ Bin Zhang, ${ }^{1}$ Na Wang, ${ }^{1}$ and Yu-hu Wang ${ }^{2}$ \\ ${ }^{1}$ Tianjin Entry-Exit Inspection and Quarantine Bureau, Tianjin 300457, China \\ ${ }^{2}$ Tianjin Lishen Battery Joint-Stock Co., Ltd., Tianjin 300384, China \\ Correspondence should be addressed to Yu-hu Wang; yuhuwang@126.com
}

Received 7 May 2013; Revised 6 June 2013; Accepted 6 June 2013

Academic Editor: A. M. S. Silva

Copyright (c) 2013 Xiao-chuan Jia et al. This is an open access article distributed under the Creative Commons Attribution License, which permits unrestricted use, distribution, and reproduction in any medium, provided the original work is properly cited.

A robust, facile, and solvent-free route for the three-component synthesis of $2 H$-indazolo[2,1- $b]$ phthalazine-triones in the presence of a catalytic amount of $p$-toluene sulfonic acid utilizing grinding has been developed. Short reaction time, simple operation, and high yields are the advantages of this protocol.

\section{Introduction}

In the past few decades, the synthesis of new heterocyclic compounds has been a subject of great interest due to their wide applicability. Among them, nitrogen heterocycles containing a phthalazine moiety are important because they show biological and pharmacological activities such as anticonvulsant, cardiotonic, and vasorelaxant [1-3]. Recently, three-component reactions of dimedone (5,5-dimethylcyclohexane-1,3-dione), an aldehyde, and phthalhydrazide to give $2 \mathrm{H}$-indazolo[2,1- $b]$ phthalazine-triones have attracted the interest of the synthetic community. Various catalytic systems including $p$-TSA [4], $\mathrm{Me}_{3} \mathrm{SiCl}$ [5], silica sulfuric acid [6], $\mathrm{H}_{2} \mathrm{SO}_{4}$ [7], cyanuric chloride [8], heteropolyacids [9], and $\mathrm{N}$-halo sulfonamides [10] have been reported. The direct four-component condensations have also been achieved by employing $\mathrm{Ce}\left(\mathrm{SO}_{4}\right)_{2} \cdot 4 \mathrm{H}_{2} \mathrm{O}$ [11], sulfuric acid-modified PEG6000 [12] under solvent-free conditions. However, some of these methods suffered from several drawbacks such as hazardous organic solvents, high cost, long reaction time, use of stoichiometric, and excess amounts of acids. Therefore, the development of a new, efficient, and environment-friendly procedure is still in demand.

Grinding method is of interest because it is performed in the absence of solvent and under environment-friendly conditions. In the last years, this technique has found interest in synthetic organic chemistry [13-15]. The reported examples include nitrone synthesis [16], Knoevenagel's reaction [17], Michael's additions [17], aldol condensation [18], coupling reactions [19], and peptide synthesis [20]. In the present work, we wish to report a versatile and green protocol for onepot, three-component synthesis of $2 H$-indazolo[2,1- $b]$ phthalazine-triones using a catalytic amount of $p$-toluene sulfonic acid (Scheme 1). The grinding is done at room temperature and the reaction time ranges from 2 to 5 minutes. This synthetic route is green as it is essentially solvent-free and involves short reaction times at ambient conditions. The workup of each reaction involves a simple washing procedure with an ethanol/water mixture. To the best of our knowledge, this is the first example to construct $2 H$-indazolo[2,1$b]$ phthalazine-trione derivatives utilizing grinding method.

\section{Results and Discussion}

We started our study by treating phthalhydrazide $(1 \mathrm{mmol})$, dimedone $(1 \mathrm{mmol})$, and benzaldehyde $(1 \mathrm{mmol})$ in a mortar and grinding them for a given time. Initially, the effect of catalyst amount on the reaction was investigated (Table 1). It was found that only $25 \%$ yield of product was obtained when grinding for 30 minutes in the absence of any catalyst (Table 1 , entry 1). Encouragingly, only $1 \mathrm{~mol} \%$ of $p$-TSA enhanced the yield up to $63 \%$ in 10 minutes. The optimization for quantity of catalyst suggested that $3 \mathrm{~mol} \%$ of $p$-TSA is enough, 


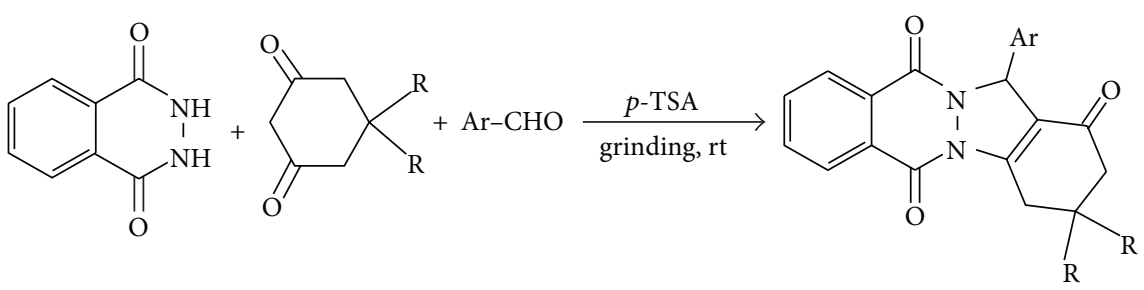

Scheme 1: Synthesis of $2 H$-indazolo[2,1-b]phthalazine-triones using grinding.

TABLE 1: Effect of catalyst amount on the reaction ${ }^{\mathrm{a}}$.

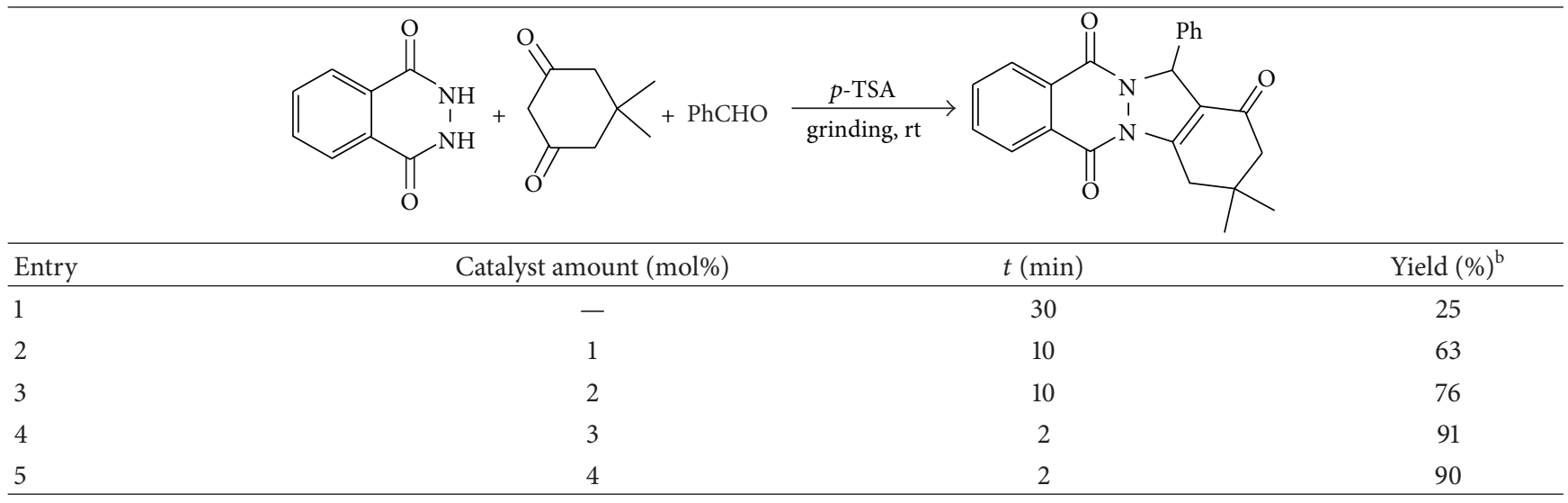

${ }^{a}$ Reaction conditions: phthalhydrazide $(1 \mathrm{mmol})$, dimedone $(1 \mathrm{mmol})$, and benzaldehyde $(1 \mathrm{mmol})$, ground at room temperature.

${ }^{\mathrm{b}}$ Isolated yields.

affording the corresponding product in $91 \%$ yield within only 2 minutes (Table 1 , entry 4 ).

The generality and functional group tolerance of this procedure in the direct synthesis of $2 \mathrm{H}$-indazolo[2,1- $b]$ phthalazine-trione derivatives were examined employing a number of substituted aromatic aldehydes under the optimized conditions (Table 2). In all cases, the starting materials became sticky and then solidified, indicating the end of the reaction. The method showed good substrate compatibility for aromatic aldehydes. Both aromatic aldehydes bearing electron-donating groups and electron-withdrawing groups gave the products in high yields. In the same way, the reaction of 1,3-cyclohexanedione for the synthesis of $2 \mathrm{H}$-indazolo[2,1$b]$ phthalazine-triones under the optimum conditions was examined and the desired products were obtained in high yields (Table 2, entries 9-12). In addition, the product could be easily purified by washing using an ethanol/water mixture $(1: 3 \mathrm{v} / \mathrm{v})$. Thus, this method offers significant improvements with regard to the scope of the transformation, simplicity, and green aspects.

To compare the advantage of our protocol with the reported procedure, the comparison results were listed in Table 3. As can be seen from the table, the reported procedures required high catalyst loading, long reaction time, and corrosive catalyst. These results clearly demonstrated that this protocol was more advantageous.
In summary, a robust, facile, and solvent-free route for the three-component synthesis of $2 \mathrm{H}$-indazolo[2,1- $b]$ phthalazine-triones in the presence of catalytic $p$-TSA using grinding has been developed. The reaction affords the products with high yields in very short reaction times. The green reaction conditions together with their simple purification process offered significant improvements over the reported methods.

\section{Experimental}

All reagents were obtained from local commercial suppliers and used without further purification. Melting points were determined on an X-4 apparatus. Analytical thin-layer chromatography was performed on glass plates of silica gel GF254 of $0.2 \mathrm{~mm}$ thickness. Mass spectra were performed on a Thermo Finnigan LCQ Advantage instrument with electrospray ionization (ESI, $4.5 \mathrm{keV}$ ) or Thermo Fisher Scientific DSQ-II instrument with electron ionization (EI, $70 \mathrm{eV}) .{ }^{1} \mathrm{H}$ and ${ }^{13} \mathrm{C}$ NMR spectra were recorded on a Bruker Advance III 500 analyzer. All the products are known compounds and were identified by comparing of their physical and spectra data with those reported in the literature.

Typical procedure for the synthesis of $2 \mathrm{H}$-indazolo[2,1$b]$-phthalazine-triones: in a mortar, a mixture of phthalhydrazide $(1 \mathrm{mmol})$, aromatic aldehyde $(1 \mathrm{mmol})$, and dimedone or 1,3-cyclohexanedione $(1 \mathrm{mmol})$ was ground together 
TABLE 2: Synthesis of $2 H$-indazolo[2,1- $b]$ phthalazine-trione derivatives under grinding.

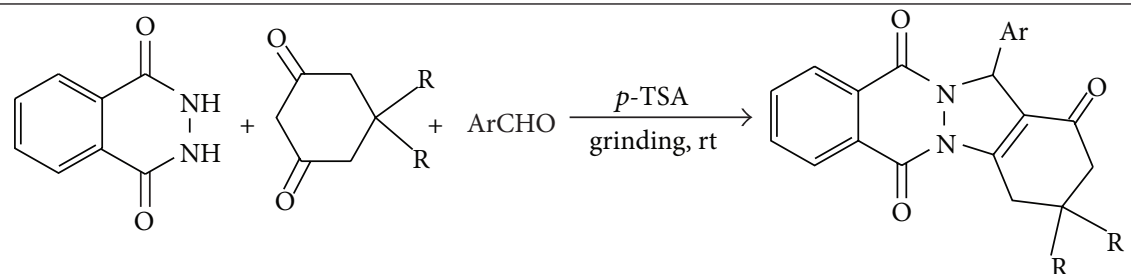

\begin{tabular}{|c|c|c|c|c|c|}
\hline Entry & $\mathrm{Ar}$ & $\mathrm{R}$ & $t(\min )$ & Product & Yield (\%) $)^{\mathrm{a}}$ \\
\hline 1 & $\mathrm{C}_{6} \mathrm{H}_{5}$ & $\mathrm{CH}_{3}$ & 2 & a & 91 \\
\hline 2 & $4-\mathrm{CH}_{3} \mathrm{C}_{6} \mathrm{H}_{4}$ & $\mathrm{CH}_{3}$ & 2 & b & 90 \\
\hline 3 & $4-\mathrm{CH}_{3} \mathrm{OC}_{6} \mathrm{H}_{4}$ & $\mathrm{CH}_{3}$ & 5 & c & 88 \\
\hline 4 & $4-\mathrm{FC}_{6} \mathrm{H}_{4}$ & $\mathrm{CH}_{3}$ & 2 & d & 87 \\
\hline 5 & $4-\mathrm{ClC}_{6} \mathrm{H}_{4}$ & $\mathrm{CH}_{3}$ & 2 & e & 86 \\
\hline 6 & $2-\mathrm{ClC}_{6} \mathrm{H}_{4}$ & $\mathrm{CH}_{3}$ & 2 & f & 83 \\
\hline 7 & $4-\mathrm{BrC}_{6} \mathrm{H}_{4}$ & $\mathrm{CH}_{3}$ & 2 & g & 85 \\
\hline 8 & $4-\mathrm{NO}_{2} \mathrm{C}_{6} \mathrm{H}_{4}$ & $\mathrm{CH}_{3}$ & 2 & $\mathbf{h}$ & 92 \\
\hline 9 & $4-\mathrm{OHC}_{6} \mathrm{H}_{4}$ & $\mathrm{H}$ & 5 & i & 86 \\
\hline 10 & $4-\left(\mathrm{CH}_{3}\right)_{2} \mathrm{NC}_{6} \mathrm{H}_{4}$ & $\mathrm{H}$ & 5 & $\mathbf{j}$ & 84 \\
\hline 11 & 2-Naphthyl & $\mathrm{H}$ & 2 & $\mathbf{k}$ & 89 \\
\hline 12 & $4-\mathrm{CH}_{3} \mathrm{C}_{6} \mathrm{H}_{4}$ & $\mathrm{H}$ & 2 & 1 & 90 \\
\hline
\end{tabular}

${ }^{\mathrm{a}}$ Isolated yields.

TABLE 3: Comparison study with reported methods.

\begin{tabular}{|c|c|c|c|c|c|}
\hline Entry & Reaction conditions & Catalyst loadings (mol\%) & $t(\min )$ & Yield (\%) & References \\
\hline 1 & p-TSA/ethanol/reflux & 10 & 360 & 47 & - \\
\hline 2 & $p$-TSA/solvent-free $/ 80^{\circ} \mathrm{C}$ & 30 & 10 & 93 & {$[4]$} \\
\hline 3 & $\mathrm{H}_{2} \mathrm{SO}_{4} /[\mathrm{bmim}]\left[\mathrm{BF}_{4}\right] / 80^{\circ} \mathrm{C}$ & 15 & 30 & 94 & [7] \\
\hline 4 & Phosphomolybdic acid- $\mathrm{SiO}_{2} / 100^{\circ} \mathrm{C}$ & 5 & 30 & 85 & {$[21]$} \\
\hline 5 & $\mathrm{CAN} / \mathrm{PEG} 400 / 50^{\circ} \mathrm{C}$ & 5 & 120 & 94 & {$[22]$} \\
\hline 6 & $p$-TSA/grinding/rt & 3 & 2 & 91 & This work \\
\hline
\end{tabular}

with a pestle. The mixture became sticky and solidified to give an orange solid, usually within $2 \sim 5$ minutes. An ethanol/water mixture $(1: 3 \mathrm{v} / \mathrm{v})(5 \mathrm{~mL})$ was then added and the mixture was ground for another $2 \mathrm{~min}$. The product was filtered, dried, and recrystallized from ethanol (if necessary).

3,4-Dihydro-3,3-dimethyl-13-phenyl-2H-indazolo[2,1b]phthalazine-1,6,11(13H)-trione (a, $\left.\mathrm{C}_{23} \mathrm{H}_{20} \mathrm{~N}_{2} \mathrm{O}_{3}\right)$ : Mp 208$209^{\circ} \mathrm{C}\left[204-206^{\circ} \mathrm{C}\right.$, [4]]; ${ }^{1} \mathrm{H} \mathrm{NMR}\left(500 \mathrm{MHz}, \mathrm{CDCl}_{3}\right) \delta=1.23$ $(\mathrm{s}, 6 \mathrm{H}), 2.35$ (s, 2H), 3.26-3.44 (m, 2H), 6.47 (s, 1H), 7.33-7.41 (m, 3H), 7.44-7.46 (m, 2H), 7.74-7.86 (m, 2H), 8.27-8.37 (m, $2 \mathrm{H}) ;{ }^{13} \mathrm{C}$ NMR $\left(125 \mathrm{MHz}, \mathrm{CDCl}_{3}\right) \delta=28.5,28.7,34.7,38.1$, 50.9, 64.9, 118.6, 127.1, 127.7, 127.9, 128.7, 128.9, 129.1, 133.6, 134.5, 136.4, 150.9, 154.3, 156.1, 192.2; MS (EI) $\mathrm{m} / z 372\left(\mathrm{M}^{+}\right)$.

3,4-Dihydro-3,3-dimethyl-13-(4-methylphenyl)- $2 \mathrm{H}$-indazolo[2,1-b]phthalazine-1,6,11(13H)-trione (b, $\mathrm{C}_{24} \mathrm{H}_{22} \mathrm{~N}_{2} \mathrm{O}_{3}$ ): $\mathrm{Mp} \quad 226-228^{\circ} \mathrm{C} \quad\left[227-229^{\circ} \mathrm{C},[4]\right] ;{ }^{1} \mathrm{H}$ NMR $(500 \mathrm{MHz}$, $\left.\mathrm{CDCl}_{3}\right) \delta=1.23(\mathrm{~s}, 6 \mathrm{H}), 2.30(\mathrm{~s}, 3 \mathrm{H}), 2.35$ (s, 2H), 3.24-3.43 $(\mathrm{m}, 2 \mathrm{H}), 6.43(\mathrm{~s}, 1 \mathrm{H}), 7.12-7.15(\mathrm{~m}, 2 \mathrm{H}), 7.31-7.38(\mathrm{~m}, 2 \mathrm{H})$, 7.83-7.87 (m, 2H), 8.28-8.38 (m, 2H); ${ }^{3} \mathrm{C}$ NMR (125 MHz, $\left.\mathrm{CDCl}_{3}\right) \delta=21.3,28.5,28.8,34.7,38.1,50.9,64.9$, 118.7, 127.1,
$127.7,127.9,128.9,129.2,129.5,133.4,133.5,134.5,138.5,150.8$, 154.2, 156.1, 192.2; MS (EI) $m / z 386\left(\mathrm{M}^{+}\right)$.

3,4-Dihydro-3,3-dimethyl-13-(4-methoxyphenyl)-2H-indazolo[2,1- $b]$ phthalazine-1,6,11(13H)-trione (c, $\mathrm{C}_{24} \mathrm{H}_{22} \mathrm{~N}_{2} \mathrm{O}_{4}$ ): $\mathrm{Mp} \quad 216-218^{\circ} \mathrm{C} \quad\left[218-220^{\circ} \mathrm{C}\right.$, [23]]; ${ }^{1} \mathrm{H}$ NMR $(500 \mathrm{MHz}$, $\left.\mathrm{CDCl}_{3}\right) \delta=1.21(\mathrm{~s}, 6 \mathrm{H}), 2.34(\mathrm{~s}, 2 \mathrm{H}), 3.23-3.42(\mathrm{~m}, 2 \mathrm{H}), 3.76$ $(\mathrm{s}, 3 \mathrm{H}), 6.42(\mathrm{~s}, 1 \mathrm{H}), 6.84-6.86(\mathrm{~m}, 2 \mathrm{H}), 7.35-7.37(\mathrm{~m}, 2 \mathrm{H})$, $7.82-7.86(\mathrm{~m}, 2 \mathrm{H}), 8.26-8.35(\mathrm{~m}, 2 \mathrm{H}) ;{ }^{13} \mathrm{C}$ NMR $(125 \mathrm{MHz}$, $\left.\mathrm{CDCl}_{3}\right) \delta=28.5,28.7,34.6,38.1,51.0,55.2,64.6,114.1,118.6$, 127.7, 127.9, 128.4, 128.5, 129.0, 129.2, 133.5, 134.5, 150.7, 154.2, 156.0, 159.7, 192.2; MS (EI) $m / z 402\left(\mathrm{M}^{+}\right)$.

3,4-Dihydro-3,3-dimethyl-13-(4-fluorophenyl)-2 $\mathrm{H}$-indazolo[2,1- $b$ ] phthalazine-1,6,11(13H)-trione (d, $\left.\mathrm{C}_{23} \mathrm{H}_{19} \mathrm{FN}_{2} \mathrm{O}_{3}\right)$ :

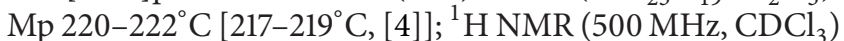
$\delta=1.21(\mathrm{~s}, 6 \mathrm{H}), 2.34(\mathrm{~s}, 2 \mathrm{H}), 3.23-3.41(\mathrm{~m}, 2 \mathrm{H}), 6.43(\mathrm{~s}, 1 \mathrm{H})$, 6.99-7.03 (m, 2H), 7.39-7.43 (m, 2H), 7.85-7.88 (m, 2H), 8.26-8.35 (m, 2H); ${ }^{13} \mathrm{C} \mathrm{NMR}\left(125 \mathrm{MHz}, \mathrm{CDCl}_{3}\right) \delta=28.5$, 28.7, 34.7, 38.0, 50.9, 64.3, 115.5, 115.9, 118.2, 127.7, 128.0, 128.9, $129.1,132.2,133.7,134.6,151.1,154.4,156.0,192.2$; MS (EI) $m / z$ $390\left(\mathrm{M}^{+}\right)$. 
3,4-Dihydro-3,3-dimethyl-13-(4-chlorophenyl)-2 $\mathrm{H}$-indazolo[2,1-b]phthalazine-1,6,11(13H)-trione $\left(\mathbf{e}, \mathrm{C}_{23} \mathrm{H}_{19} \mathrm{ClN}_{2} \mathrm{O}_{3}\right)$ : $\mathrm{Mp} 262-264^{\circ} \mathrm{C} \quad\left[262-264^{\circ} \mathrm{C},[4]\right] ;{ }^{1} \mathrm{H}$ NMR $(500 \mathrm{MHz}$, $\left.\mathrm{CDCl}_{3}\right) \delta=1.22(\mathrm{~s}, 3 \mathrm{H}), 1.23(\mathrm{~s}, 3 \mathrm{H}), 2.35$ (s, 2H), 3.25-3.43 $(\mathrm{m}, 2 \mathrm{H}), 6.43(\mathrm{~s}, 1 \mathrm{H}), 7.31-7.33(\mathrm{~m}, 2 \mathrm{H}), 7.37-7.41(\mathrm{~m}, 2 \mathrm{H})$, 7.85-7.88 (m, 2H), 8.26-8.39 (m, 2H); ${ }^{3} \mathrm{C}$ NMR $(125 \mathrm{MHz}$, $\left.\mathrm{CDCl}_{3}\right) \delta=28.5,28.7,34.7,38.0,50.9,64.3,118.1,127.7,128.1$, $128.5,128.8,128.9,129.0,133.7,134.5,134.6,134.9,151.1,154.3$, 156.0, 192.2; MS (EI) $\mathrm{m} / z 406\left(\mathrm{M}^{+}\right)$.

3,4-Dihydro-3,3-dimethyl-13-(2-chlorophenyl)-2 $\mathrm{H}$-indazolo[2,1-b] phthalazine-1,6,11(13H)-trione $\left(\mathbf{f}, \mathrm{C}_{23} \mathrm{H}_{19} \mathrm{ClN}_{2} \mathrm{O}_{3}\right)$ : $\mathrm{Mp} \quad 268-270^{\circ} \mathrm{C}\left[264-266^{\circ} \mathrm{C},[4]\right] ;{ }^{1} \mathrm{H}$ NMR $(500 \mathrm{MHz}$, $\left.\mathrm{CDCl}_{3}\right) \delta=1.22(\mathrm{~s}, 3 \mathrm{H}), 1.23(\mathrm{~s}, 3 \mathrm{H}), 2.33(\mathrm{~s}, 2 \mathrm{H}), 3.24-3.42$ $(\mathrm{m}, 2 \mathrm{H}), 6.69(\mathrm{~s}, 1 \mathrm{H}), 7.25-7.34(\mathrm{~m}, 2 \mathrm{H}), 7.49-7.50(\mathrm{~m}, 2 \mathrm{H})$, 7.84-7.89 (m, 2H), 8.25-8.40 (m, 2H); ${ }^{13} \mathrm{C}$ NMR $(125 \mathrm{MHz}$, $\left.\mathrm{CDCl}_{3}\right) \delta=28.4,28.8,34.6,38.0,50.9,64.0,116.7,127.2,127.6$, $128.0,128.7,129.0,129.9,130.5,132.6,133.0,133.6,134.5,151.9$, 154.2, 156.2, 192.1; MS (EI) $m / z 406\left(\mathrm{M}^{+}\right)$.

3,4-Dihydro-3,3-dimethyl-13-(4-bromophenyl)-2H-indazolo[2,1-b] phthalazine-1,6,11(13H)-trione (g, $\left.\mathrm{C}_{23} \mathrm{H}_{19} \mathrm{BrN}_{2} \mathrm{O}_{3}\right)$ : $\mathrm{Mp} \quad 264-266^{\circ} \mathrm{C}\left[265-267^{\circ} \mathrm{C}\right.$, [4]]; ${ }^{1} \mathrm{H}$ NMR $(500 \mathrm{MHz}$, $\left.\mathrm{CDCl}_{3}\right) \delta=1.21(\mathrm{~s}, 3 \mathrm{H}), 1.22(\mathrm{~s}, 3 \mathrm{H}), 2.35(\mathrm{~s}, 2 \mathrm{H}), 3.24-3.41$ $(\mathrm{m}, 2 \mathrm{H}), 6.41(\mathrm{~s}, 1 \mathrm{H}), 7.29-7.31(\mathrm{~m}, 2 \mathrm{H}), 7.35-7.38(\mathrm{~m}, 2 \mathrm{H})$, $7.82-7.84(\mathrm{~m}, 2 \mathrm{H}), 8.27-8.38(\mathrm{~m}, 2 \mathrm{H}) ;{ }^{13} \mathrm{C}$ NMR $(125 \mathrm{MHz}$, $\left.\mathrm{CDCl}_{3}\right) \delta=28.5,28.7,34.7,38.0,50.9,64.4,118.0,122.8,127.8$, $128.1,128.8,128.9,129.0,131.9,133.7,134.7,135.5,151.1,154.4$, 156.0, 192.1; MS (EI) $m / z 451\left(\mathrm{M}^{+}\right)$.

3,4-Dihydro-3,3-dimethyl-13-(4-nitrophenyl)- $2 \mathrm{H}$-indazolo[2,1-b]phthalazine-1,6,11(13H)-trione (h, $\left.\mathrm{C}_{23} \mathrm{H}_{19} \mathrm{~N}_{3} \mathrm{O}_{5}\right)$ : $\mathrm{Mp} \quad 220-222^{\circ} \mathrm{C} \quad\left[223-225^{\circ} \mathrm{C},[4]\right] ;{ }^{1} \mathrm{H}$ NMR $(500 \mathrm{MHz}$, $\left.\mathrm{CDCl}_{3}\right) \delta=1.21(\mathrm{~s}, 3 \mathrm{H}), 1.23(\mathrm{~s}, 3 \mathrm{H}), 2.33-2.38(\mathrm{~m}, 2 \mathrm{H})$, 3.26-3.43 (m, 2H), 6.52 (s, 1H), 7.62-7.64 (m, 2H), $7.90(\mathrm{~m}$, $2 \mathrm{H}), 8.21(\mathrm{~m}, 2 \mathrm{H}), 8.24-8.41(\mathrm{~m}, 2 \mathrm{H}) ;{ }^{13} \mathrm{C} \mathrm{NMR}(125 \mathrm{MHz}$, $\left.\mathrm{CDCl}_{3}\right) \delta=28.4,28.7,34.7,38.0,50.8,64.2,117.3,124.1,127.8$, 128.1, 128.3, 128.6, 128.9, 133.9, 134.9, 143.4, 147.9, 151.7, 154.6, 155.9, 192.1; MS (EI) $m / z 417\left(\mathrm{M}^{+}\right)$.

3,4-Dihydro-13-(4-hydroxyphenyl)- $2 \mathrm{H}$-indazolo [2,1b]phthalazine-1,6,11(13H)-trione $\left(\mathrm{i}, \mathrm{C}_{23} \mathrm{H}_{20} \mathrm{~N}_{2} \mathrm{O}_{4}\right)$ : Mp 258$260^{\circ} \mathrm{C}\left[265-266^{\circ} \mathrm{C},[7]\right] ;{ }^{1} \mathrm{H}$ NMR $\left(500 \mathrm{MHz}, \mathrm{CDCl}_{3}\right) \delta=$ 2.24-2.28 (m, 2H), 2.46-2.47 (m, 2H), 3.35-3.60 (m, 2H), $6.37(\mathrm{~s}, 1 \mathrm{H}), 6.78(\mathrm{~d}, 2 \mathrm{H}), 7.23(\mathrm{~d}, 2 \mathrm{H}), 7.83-7.86(\mathrm{~m}, 2 \mathrm{H})$, 8.23-8.35 (m, 2H), 8.83 (s, $1 \mathrm{H}) ;{ }^{13} \mathrm{C} \mathrm{NMR}\left(125 \mathrm{MHz} \mathrm{CDCl}_{3}\right.$ ) $\delta=27.0,29.2$, 41.7, 69.3, 120.3, 124.2, 130.3, 131.8, 132.1, 132.7, $133.4,133.7,137.1,138.3,139.3,157.1,158.8,160.6,162.4,197.3$; MS (EI) $m / z 360\left(\mathrm{M}^{+}\right)$.

3,4-Dihydro-13-(4-(dimethylamino) phenyl)-2 $\mathrm{H}$-indazolo[2, 1- $b$ ] phthalazine-1,6,11(13H)-trione $\left(\mathbf{j}, \mathrm{C}_{25} \mathrm{H}_{25} \mathrm{~N}_{3} \mathrm{O}_{3}\right)$ : $\mathrm{Mp} \quad 258-260^{\circ} \mathrm{C} \quad\left[256-258^{\circ} \mathrm{C}\right.$, [7]]; ${ }^{1} \mathrm{H}$ NMR $(500 \mathrm{MHz}$, $\left.\mathrm{CDCl}_{3}\right) \delta=2.24-2.28(\mathrm{~m}, 2 \mathrm{H}), 2.46-2.48(\mathrm{~m}, 2 \mathrm{H}), 2.89(\mathrm{~s}$, $6 \mathrm{H}), 3.35-3.61(\mathrm{~m}, 2 \mathrm{H}), 6.40(\mathrm{~s}, 1 \mathrm{H}), 6.63-6.66(\mathrm{~m}, 2 \mathrm{H}), 7.26-$ 7.29 (m, 2H), 7.80-7.83 (m, 2H), 8.25-8.34 (m, 2H); ${ }^{13} \mathrm{C} \mathrm{NMR}$ $\left(125 \mathrm{MHz} \mathrm{CDCl}_{3}\right) \delta=22.4,24.5,37.0,40.3,40.6,64.8,112.2$, $112.4,119.9,123.4,127.7,127.8,128.2,128.9,129.0,129.3,133.2$, 134.3, 150.6, 151.8, 154.1, 156.1, 192.5; MS (EI) $m / z 387\left(\mathrm{M}^{+}\right)$.

3,4-Dihydro-13-(naphthalen-2-yl)- $2 \mathrm{H}$-indazolo [2,1b]phthalazine-1,6,11(13H)-trione $\left(\mathbf{k}, \mathrm{C}_{25} \mathrm{H}_{18} \mathrm{~N}_{2} \mathrm{O}_{3}\right)$ : Mp 261$262^{\circ} \mathrm{C}\left[262-264^{\circ} \mathrm{C},[7]\right] ;{ }^{1} \mathrm{H}$ NMR $\left(500 \mathrm{MHz} \mathrm{CDCl}_{3}\right) \delta=$ $2.21-2.29(\mathrm{~m}, 2 \mathrm{H}), 2.43-2.47(\mathrm{~m}, 2 \mathrm{H}), 3.28-3.65(\mathrm{~m}, 2 \mathrm{H}), 6.60$ (s, $1 \mathrm{H}), 7.41-7.50(\mathrm{~m}, 3 \mathrm{H}), 7.75-7.91(\mathrm{~m}, 6 \mathrm{H}), 8.20-8.38(\mathrm{~m}$,
$2 \mathrm{H}) ;{ }^{13} \mathrm{C}$ NMR $\left(125 \mathrm{MHz}, \mathrm{CDCl}_{3}\right) \delta=22.3,24.5,36.9,65.1$, $119.7,124.3,126.2,126.3,126.7,127.6,127.7,128.0,128.2,128.6$, $129.0,129.1,133.2,133.4,133.5,133.6,134.5,152.3,154.3,156.1$, 192.4; MS (EI) $m / z 394\left(\mathrm{M}^{+}\right)$.

3,4-Dihydro-13-( $p$-tolyl)-2H-indazolo[2,1- $b$ ]phthalazine-1, 6,11(13H)-trione (l, $\left.\mathrm{C}_{22} \mathrm{H}_{18} \mathrm{~N}_{2} \mathrm{O}_{3}\right)$ : $\mathrm{Mp} 244-246^{\circ} \mathrm{C}$ [248$\left.250^{\circ} \mathrm{C},[23]\right] ;{ }^{1} \mathrm{H}$ NMR $\left(500 \mathrm{MHz} \mathrm{CDCl}_{3}\right) \delta=2.23-2.27(\mathrm{~m}$, $2 \mathrm{H}), 2.29(\mathrm{~s}, 3 \mathrm{H}), 2.44-2.48(\mathrm{~m}, 2 \mathrm{H}), 3.29-3.60(\mathrm{~m}, 2 \mathrm{H}), 6.42$ (s, 1H), 7.12-7.32 (m, 4H), 7.81-7.87 (m, 2H), 8.25-8.36 (m, $2 \mathrm{H}) ;{ }^{13} \mathrm{C} \mathrm{NMR}\left(125 \mathrm{MHz}, \mathrm{CDCl}_{3}\right) \delta=21.2,22.3,24.5,36.9$, $64.8,119.8,127.1,127.7,127.9,129.0,129.2,129.4,133.3,133.4$, $134.5,138.5,152.1,154.2,156.1,192.5$; MS (EI) $m / z 358\left(\mathrm{M}^{+}\right)$.

\section{Acknowledgment}

The authors want to thank Lei Zhou and Yan Yu for their analysis of the characterization data of the compounds.

\section{References}

[1] X. Y. Sun, C. X. Wei, X. Q. Deng, Z. G. Sun, and Z. S. Quan, "Evaluation of the anticonvulsant activity of 6-(4-chlorophenyoxy)-tetrazolo[5,1-a]phthalazine in various experimental seizure models in mice," Pharmacological Reports, vol. 62, no. 2, pp. 273-277, 2010.

[2] M. Asif, "Some recent approaches of biologically active substituted pyridazine and phthalazine drugs," Current Medicinal Chemistry, vol. 19, no. 18, pp. 2984-2991, 2012.

[3] F. M. Awadallah, W. I. EI-Eraky, and D. O. Saleh, "Synthesis, vasorelaxant activity, and molecular modeling study of some new phthalazine derivatives," European Journal of Medicinal Chemistry, vol. 52, pp. 14-21, 2012.

[4] M. Sayyafi, M. Seyyedhamzeh, H. R. Khavasi, and A. Bazgir, "One-pot, three-component route to $2 \mathrm{H}$-indazolo[2,1- $b]$ phthalazine-triones," Tetrahedron, vol. 64, no. 10, pp. 2375-2378, 2008.

[5] L. Nagarapu, R. Bantu, and H. B. Mereyala, “TMSCl-mediated one-pot, three-component synthesis of $2 \mathrm{H}$-indazolo[2,1b] phthalazine-triones," Journal of Heterocyclic Chemistry, vol. 46, no. 4, pp. 728-731, 2009.

[6] H. R. Shaterian, M. Ghashang, and M. Feyzi, "Silica sulfuric acid as an efficient catalyst for the preparation of $2 \mathrm{H}$-indazolo[2,1b]phthalazine-triones," Applied Catalysis A, vol. 345, no. 2, pp. 128-133, 2008.

[7] J. M. Khurana and D. Magoo, "Efficient one-pot syntheses of $2 \mathrm{H}$-indazolo[2,1- $b$ ] phthalazine-triones by catalytic $\mathrm{H}_{2} \mathrm{SO}_{4}$ in water-ethanol or ionic liquid," Tetrahedron Letters, vol. 50, no. 52, pp. 7300-7303, 2009.

[8] X. Wang, W. W. Ma, L. Q. Wu, and F. L. Yan, "Synthesis of $2 \mathrm{H}$ indazolo[2,1-b]phthalazine-1,6,11(13H)-trione derivatives using wet cyanuric chloride under solvent-free condition," Journal of the Chinese Chemical Society, vol. 57, pp. 1341-1345, 2010.

[9] H. J. Wang, X. N. Zhang, and Z. H. Zhang, "Highly efficient three-component synthesis of $1 H$-indazolo[1,2- $b]$ phthalazinetrione derivatives catalyzed by heteropolyacids," Monatshefte für Chemie, vol. 141, no. 4, pp. 425-430, 2010.

[10] R. Ghorbani-Vaghei, R. Karimi-Nami, Z. Toghraei-Semiromi, M. Amiri, and M. Ghavidel, "One-pot synthesis of aliphatic and aromatic $2 \mathrm{H}$-indazolo[2,1-b]phthalazine- triones catalyzed by $N$-halosulfonamides under solvent-free conditions," Tetrahedron, vol. 67, no. 10, pp. 1930-1937, 2011. 
[11] E. Mosaddegh and A. Hassankhani, "A rapid, one-pot, fourcomponent route to $2 \mathrm{H}$-indazolo[2,1-b]phthalazine-triones," Tetrahedron Letters, vol. 52, no. 4, pp. 488-490, 2011.

[12] A. Hasaninejed, M. R. Kazerooni, and A. Zare, "Solventfree, one-pot, four-component synthesis of $2 \mathrm{H}$-indazolo[2,1b]phthalazine-triones using sulfuric acid-modified PEG-6000 as a green recyclable and biodegradable polymeric catalyst," Catalysis Today, vol. 196, no. 1, pp. 148-155, 2012.

[13] G. Kaupp, "Organic solid-state reactions with $100 \%$ yield," Topics in Current Chemistry, vol. 254, pp. 95-183, 2005.

[14] B. Rodrìguez, A. Bruckmann, T. Rantanen, and C. Bolm, "Solvent-free carbon-carbon bond formations in ball mills," Advanced Synthesis and Catalysis, vol. 349, no. 14-15, pp. 22132233, 2007.

[15] A. Bruckmann, A. Krebs, and C. Bolm, "Organocatalytic reactions: effects of ball milling, microwave and ultrasound irradiation," Green Chemistry, vol. 10, no. 11, pp. 1131-1141, 2008.

[16] E. Colacino, P. Nun, F. M. Colacino, J. Martinez, and F. Lamaty, "Solvent-free synthesis of nitrones in a ball-mill," Tetrahedron, vol. 64, no. 23, pp. 5569-5576, 2008.

[17] G. Kaupp, M. R. Naimi-Jamal, and J. Schmeyers, "Solvent-free Knoevenagel condensations and Michael additions in the solid state and in the melt with quantitative yield," Tetrahedron, vol. 59, no. 21, pp. 3753-3760, 2003.

[18] C. L. Raston and J. L. Scott, "Chemoselective, solvent-free aldol condensation reaction," Green Chemistry, vol. 2, no. 2, pp. 4952, 2000.

[19] D. A. Fulmer, W. C. Shearouse, S. T. Medonza, and J. MacK, "Solvent-free Sonogashira coupling reaction via high speed ball milling," Green Chemistry, vol. 11, no. 11, pp. 1821-1825, 2009.

[20] V. Declerck, P. Nun, J. Martinez, and F. Lamaty, "Solvent-free synthesis of peptides," Angewandte Chemie, vol. 48, no. 49, pp. 9318-9321, 2009.

[21] G. Sabitha, C. Srinivas, A. Raghavendar, and J. S. Yadav, "Phosphomolybdic acid (PMA)- $\mathrm{SiO}_{2}$ as a heterogeneous solid acid catalyst for the one-pot synthesis of $2 \mathrm{H}$-indazolo[1,2- $b$ ]phthalazine-triones," Helvetica Chimica Acta, vol. 93, no. 7, pp. 13751380, 2010.

[22] K. Mazaahir, C. Ritika, and J. Anwar, "Efficient CAN catalyzed synthesis of $1 H$-indazolo[1,2-b]phthalazine-1,6,11-triones: an eco-friendly protocol," Chinese Science Bulletin, vol. 57, no. 18, pp. 2273-2279, 2012.

[23] G. Shukla, R. K. Verma, G. K. Verma, and M. S. Singh, "Solventfree sonochemical one-pot three-component synthesis of $2 \mathrm{H}$ indazolo[2,1- $b]$ phthalazine-1,6,11-triones and $1 H$-pyrazolo[1,2b]phthalazine- 5,10-diones," Tetrahedron Letters, vol. 52, no. 52, pp. 7195-7198, 2011. 

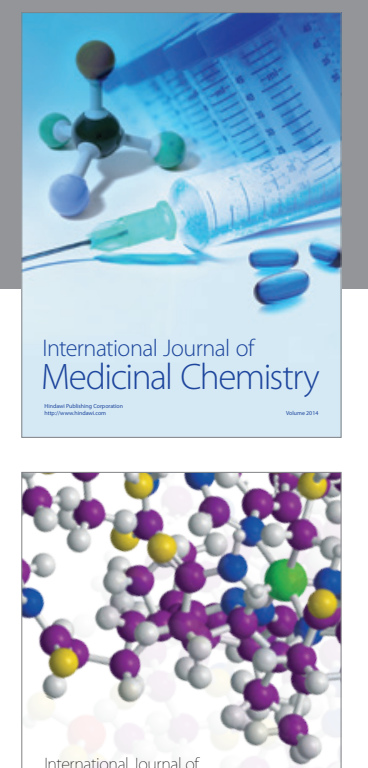

\section{Carbohydrate} Chemistry

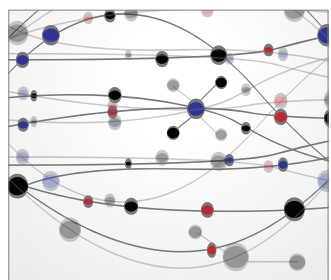

The Scientific World Journal
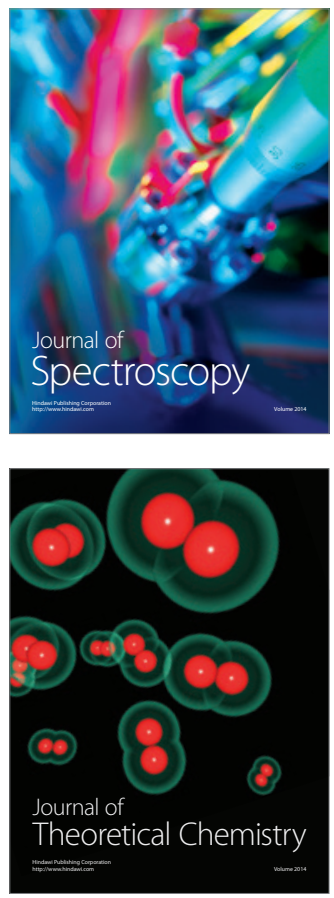
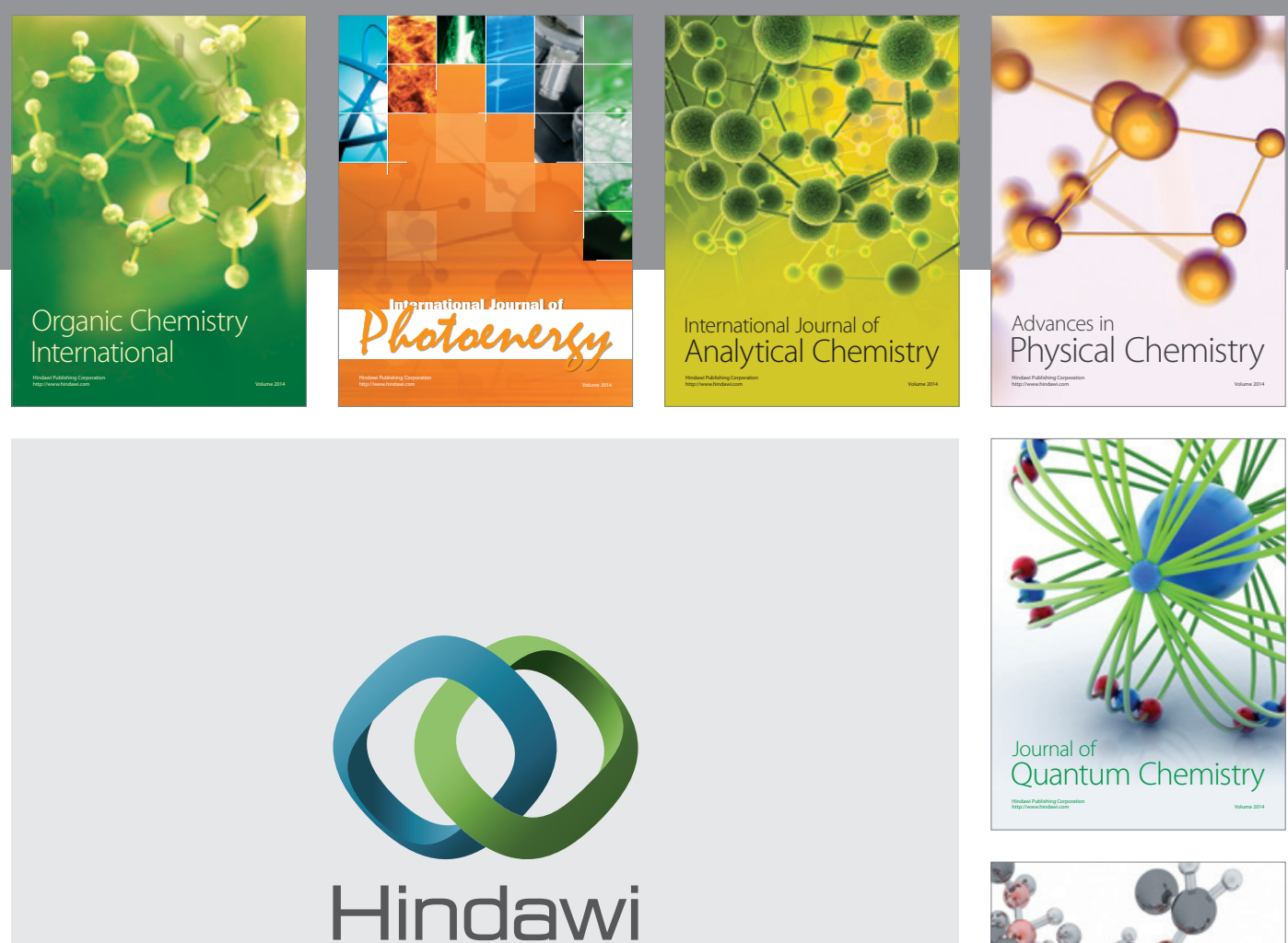

Submit your manuscripts at

http://www.hindawi.com

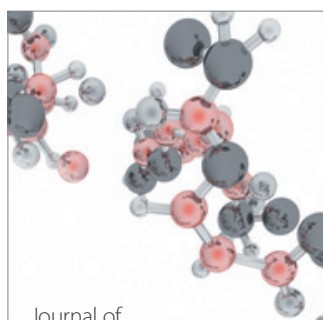

Analytical Methods

in Chemistry

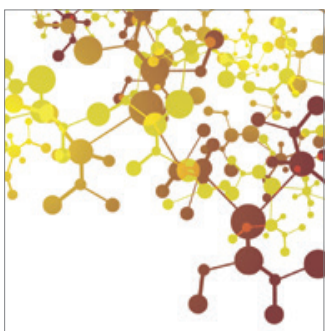

Journal of

Applied Chemistry

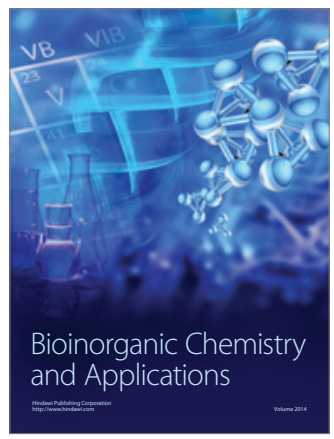

Inorganic Chemistry
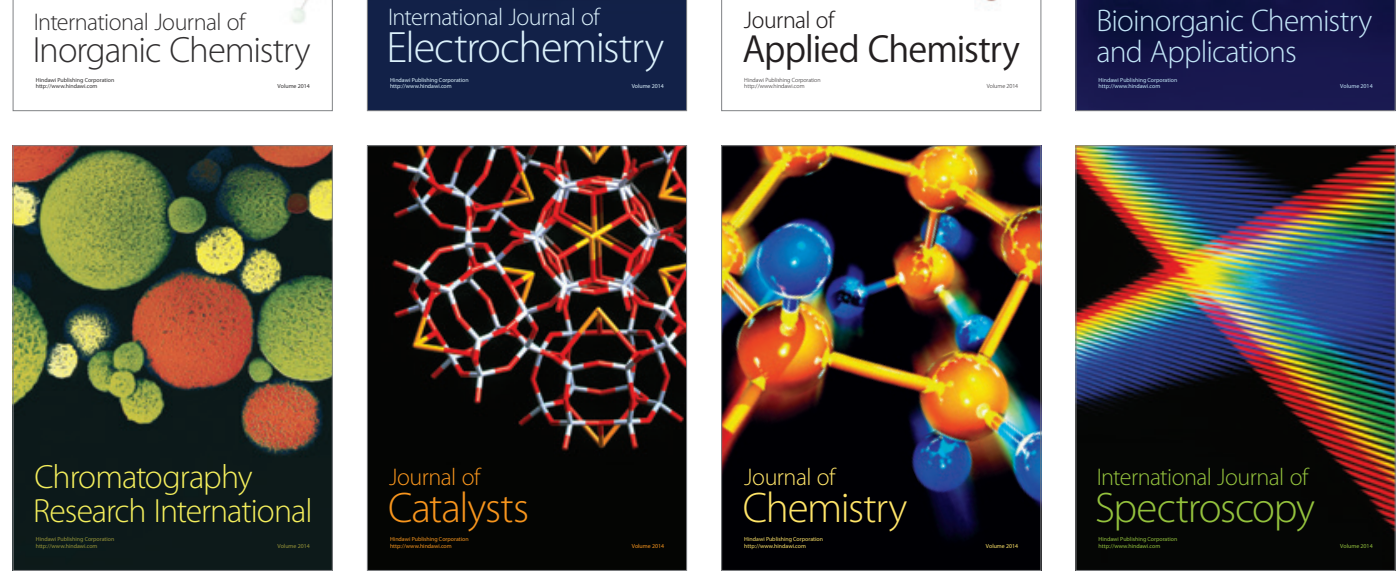